\title{
Unification of Electromagnetic Force and Gravity through the Composite Photon
}

\author{
Ian Clague M.A. Hons. (Cantab.)
}

\begin{abstract}
A deep relationship is identified between the Coulomb Force and Gravity. A gravitational constant for strong gravity is calculated from the relationship. The equivalence between mass and charge is explored. Implications are given for the expansion of Einstein's Field Equations to include vector gravity.
\end{abstract}

Keywords: Unification, Composite Photon, Negative Mass, Antimatter, Grand Unification Theory

Contact: ian.clague@sedarainv.com

Current Affiliation: Independent

\section{Introduction}

The question of whether the photon is an elementary particle or composite has been a matter of debate for almost 100 years since Louis De Broglie published his paper, A Tentative Theory of Light Quanta in 1924'. De Broglie wrote "Naturally, the light quantum must have an internal binary symmetry".

The composite theory is more descriptive of reality than the elementary theory. Perkins $(2014)^{2}$ finds that the composite theory predicts the Maxwell equations, while the elementary photon has been created to reflect them. He continues "In the elementary theory, it is difficult to describe the electromagnetic field with the four-component vector potential. This is because the photon has only two polarisation states. This problem does not exist with the composite photon theory."

Gauthier $(2019)^{3}$ has done extensive work in this area and elaborates a composite model consisting of an electron-positron pair spinning around each other in helical motion. He finds that the parameters of energy, frequency, wavelength and helical radius of each spin-1/2, halfphoton composing the double-helix photon remain the same in the transformation of the halfphotons into the relativistic electron and positron quantum vortex models.

Villata $(2011)^{4}$ has transformed matter into antimatter in the equations of both electrodynamics and gravitation. Starting from the CPT invariance of physical laws, in the former case, the result is the well-known change of sign of the electric charge. In the latter, he finds that the gravitational interaction between matter and antimatter is a mutual repulsion, i.e. antigravity appears as a prediction of general relativity when CPT is applied. This result supports cosmological models attempting to explain the accelerated expansion of the Universe in terms of a matter-antimatter repulsive interaction.

Using the work of Bondi (1957) $)^{5}$, we may interpret this finding as negative mass of the only type compatible with general relativity. The interactions of such negative mass are given in Figure 1: 


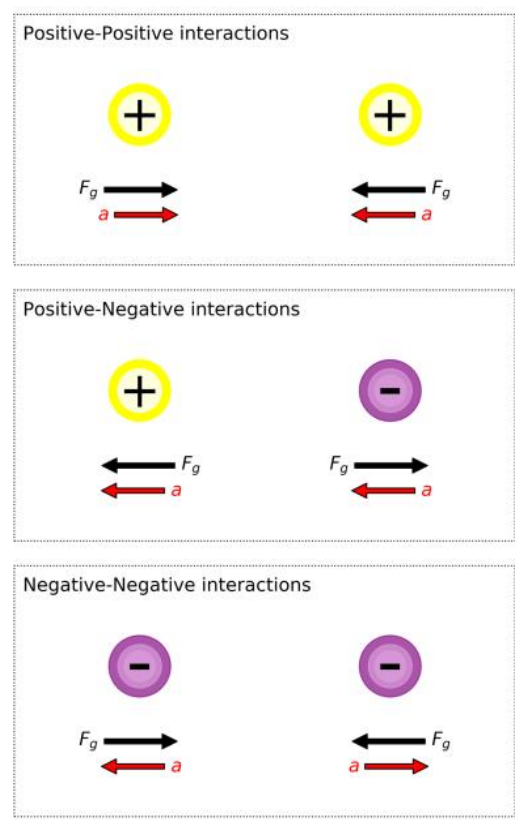

Figure 1.

For the negative mass, the acceleration is in the opposite direction to the gravitational force.

\section{Experimental Evidence}

For experimental confirmation of the photon's composition as two symmetrical half-photons, one of positive mass and one of negative mass, we can look to Wimmer, Regensberger et al. $(2103)^{6}$. They find symmetrical halves of negative and positive mass on a dispersion diagram for light pulses interacting (Figure 2). The laser pulses also display runaway self-acceleration which is expected from Figure 1. for the positive-negative mass interaction in which the accelerations of the two masses are in the same direction (Figure 3).

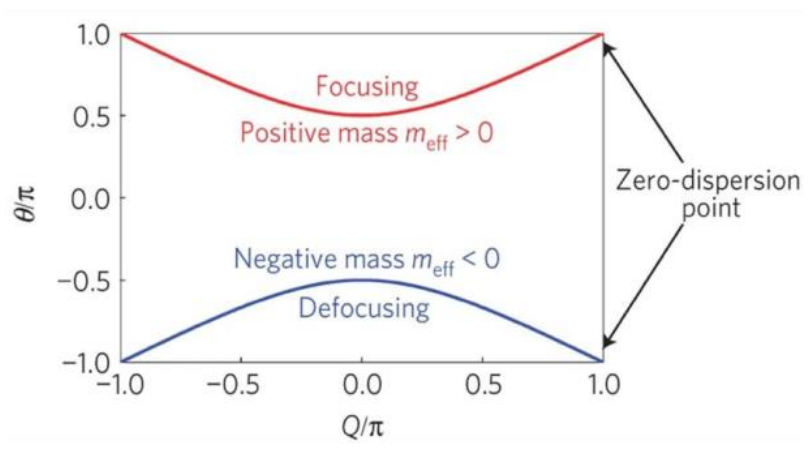

Figure 2. 


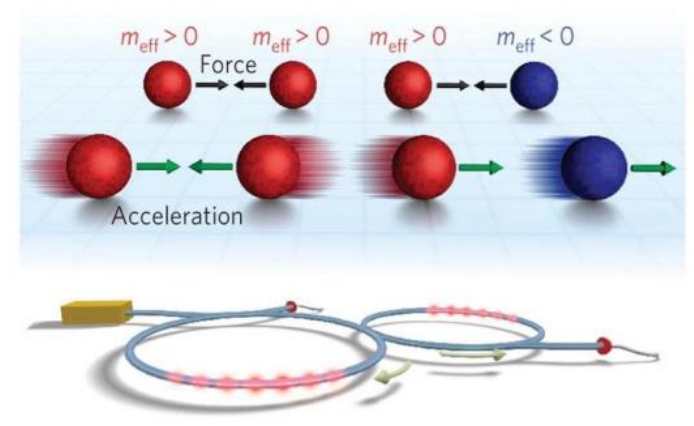

Figure 3.

That the photon consists of an electron with positive and a positron with negative mass explains why the rest mass of the photon is zero. Runaway motion between positive and negative mass explains why photons always travel at light speed.

In the absence of an electrical field, the defocusing behaviour of positron beams is evidence of the negative mass to negative mass interaction. Negative masses accelerate away from each other.

In addition, the elliptical polarization of light is experimental evidence for the composite photon. This shows the electromagnetic field to be a 4-vector. The elementary photon theory predicts only two states of (circular) polarization.

\section{Charges Follow a Potential Gradient}

We do not find, however, that the positron of negative mass will react inversely to the electromagnetic force. This would be inconsistent with experimental evidence for the electromagnetic interaction of antimatter (Gabrielse et al. 1999) ${ }^{7}$. There is no gravitational potential gradient in spectroscopy experiments to determine the mass/charge ratio of antimatter particles. Since negative mass was completely unexpected, the experimental set-up, which is largely unchanged since 1897, was not designed to detect it.

\section{Equality of Forces acting on the Electron-Positron Pair}

We now investigate the forces acting on the electron-positron pair. The centripetal force is equal to the Coulomb force acting between the negatively charged electron and the positively charged positron. In addition, from the arguments above, a repulsive gravitational force between the matter and antimatter particles is equal to the attractive Coulomb force. We have: 
For two half-photons separated by a distance $D=\frac{\lambda}{\pi} \quad$ where $\lambda=$ wavelength of the photon

$$
\begin{aligned}
& F_{\text {centripetal }}=2 \pi \frac{c}{\lambda} \cdot \frac{1}{2} \cdot \frac{h}{\lambda} \\
& =\frac{\pi c h}{\lambda^{2}} \\
& =\frac{\pi c h}{D^{2} \pi^{2}} \quad \lambda=\pi D \\
& =\frac{c h}{D^{2} \pi} \\
& =\frac{G m_{e} m_{p}}{D^{2}}=F_{\text {gravitational }} \quad m_{p=\text { mass of positron }} \\
& G=\frac{c h}{\pi m_{e}^{2}} \\
& G_{s}=\frac{2 \hbar c}{m_{e}^{2}} \quad \text { This is the Gravitational Constant for strong gravity }
\end{aligned}
$$

This compares to:

$$
G=\frac{\hbar c}{\mathrm{M}_{\mathrm{p}}^{2}} \quad \text { For Newton's Gravitational Constant, where } \mathrm{M}_{\mathrm{p}}=\text { Planck mass }
$$

This indicates the existence of a strong version of the gravitational force operating inside the composite photon consisting of an electron-positron pair.

This strong gravitational force is stronger than the Newtonian gravitational force in the ratio:

$$
G_{s} /_{G}=\frac{2 \mathrm{M}_{\mathrm{p}}{ }^{2}}{m_{e}{ }^{2}} \quad \text { or } 45 \text { orders of magnitude stronger than the Newtonian gravitational force }
$$

For consistency, we check:

$$
\begin{aligned}
F_{\text {Coulomb }} & =F_{\text {gravitational }} \\
\frac{Q^{2}}{4 \pi \varepsilon_{0} D^{2}} & =\frac{G_{S} m_{e}^{2}}{D^{2}} \\
G_{S} & =2 e^{2} / 4 \pi \varepsilon_{0} \alpha m_{e}^{2} \\
& =\frac{2 \hbar c}{m_{e}^{2}}
\end{aligned}
$$

where $\mathrm{Q}=\mathrm{e} \sqrt[2]{2 / \alpha}=16.6 \mathrm{e}$ is the magnitude of the charge on each helically-moving half-photon$$
\alpha=\text { fine structure constant }
$$$$
\varepsilon_{0}=\text { permittivity of the vacuum }
$$

since $e^{2}=4 \pi \varepsilon_{0} \hbar c \alpha$

This confirms the value of the strong gravitational constant, $G_{S}$, so the gravitational force becomes equal to the Coulomb force for $G_{s}=\frac{2 \hbar c}{m_{e}^{2}}$. Note that the value of $G_{s}$ is independent of 
the wavelength of the photon. The strong gravitational force acts on all photons, regardless of their energy.

This provides a unification between the electromagnetic force and the gravitational force, at least in the case of the electron-positron pair.

\section{Is this truly a Unification or simply an Equivalence?}

$$
\begin{aligned}
F_{\text {Coulomb }} & =F_{\text {gravitational }} \\
\frac{Q^{2}}{4 \pi \varepsilon_{0} D^{2}} & =\frac{G_{s} m_{e}^{2}}{D^{2}} \\
\frac{2 e^{2}}{4 \pi \alpha \varepsilon_{0} D^{2}} & =\frac{G_{s} m_{e}^{2}}{D^{2}} \\
\frac{2 e^{2}}{G_{S} D^{2}} & =\frac{m_{e}{ }^{2} \cdot 4 \pi \alpha \varepsilon_{0}}{D^{2}}
\end{aligned}
$$

where $\mathrm{Q}=\mathrm{e} \sqrt[2]{2 / \alpha}=16.6 \mathrm{e}$ is the magnitude of the charge on each helically-moving half-photon $\alpha=$ fine structure constant $\varepsilon_{0}=$ permittivity of the vacuum

In this representation, we have an electromagnetic force with a gravitational constant is equivalent to strong gravity with an electromagnetic constant.

The two forces are different aspects of the same force, one attractive and the other repulsive.

\section{Consequences}

The electromagnetic force unifies with the strong gravitational force present in composite photons consisting of an electron-positron pair. This strong gravitational force is 45 orders of magnitude stronger than Newtonian gravity.

This unification provides a framework for the unification of the four fundamental forces of nature since the weak force, electromagnetic force and strong force have already been shown to unify. It provides a potential resolution to the Hierarchy Problem of why Newtonian gravity is so much weaker than the other forces.

The composite photon model developed by Gauthier ${ }^{3}$ and augmented here give some deep insights into the process of transformation of light into matter and antimatter and the annihilation process of matter and antimatter into photons.

\section{Observational Evidence for Antimatter having Negative Mass}

Composite photons consisting of particle-antiparticle pairs having positive and negative mass provide a physical interpretation at the level of particle physics for the Pair Creation Model of the Universe developed by Choi and Rudra $(2104)^{8}$. This gives, for the first time, a fully consistent and lucid explanation of how the universe developed from net zero energy and evolved into the distribution of energy density we observe today. 
Indeed, the results of Choi and Rudra's ${ }^{8}$ simulation correspond closely with observations:

\begin{tabular}{|c|c|c|c|}
\hline \multicolumn{4}{|c|}{ Energy Distribution in the Universe } \\
\hline & WMAP & Simulation & Planck \\
\hline Matter & 4.6 & 4.5 & 4.9 \\
\hline Dark Matter & 23.3 & 25.1 & 26.8 \\
\hline Dark Energy & 72.1 & 70.3 & 68.3 \\
\hline
\end{tabular}

Composite photons consisting of particle-antiparticle pairs having positive and negative mass also provide a physical interpretation at the level of particle physics for the gravitational dipoles proposed by Hadjukovic ${ }^{9}$. Support is also given to negative mass cosmologies developed by J. S. Farnes ${ }^{10}$ and Choi and Rudra ${ }^{8}$ which correspond well to observational evidence of the interactions and behaviour of Dark Matter and Dark Energy. The composite photon development given here thus benefits from the same observational evidence which must be contrasted with the absolute failure of experiments to detect Dark Matter particles or Dark Energy in the laboratory.

\section{Gravity in the Early Universe}

Strong gravitational force $=\frac{G_{s} m_{e}^{2}}{D^{2}}=\frac{2 h c}{2 \pi D^{2}} \quad$ for the electron-positron pair (the elementary charged particles).

This follows an inverse square law but is independent of the Gravitational constant. It tends to a maximum value of $F_{\max }=\frac{2 h c}{2 \pi \ell_{P}^{2}}$ as the distance between the electron and positron tends to the Planck length and is repulsive.

$$
\begin{aligned}
& F_{\max }=\frac{2 h c}{2 \pi \ell_{P}{ }^{2}}=\frac{2 \hbar c}{\hbar G} \cdot c^{3} \quad \text { where } \ell_{P}=\text { Plank length and since } \ell_{P}{ }^{2}=\frac{\hbar G}{c^{3}} \\
& F_{\max }=\frac{2 c^{4}}{G} \quad \text { which is } 2 \text { times the Planck force }
\end{aligned}
$$




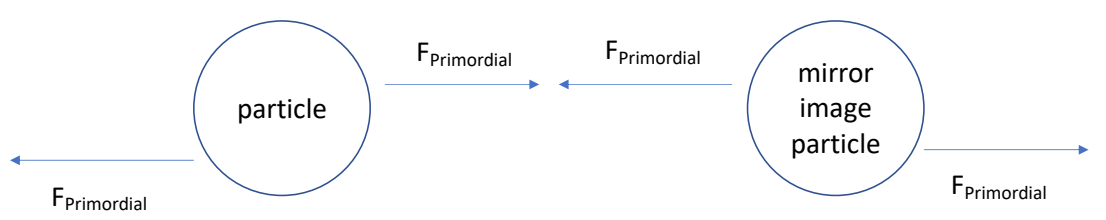

Figure 4.

Figure 4 presents a picture of the primordial force in the early universe. One force is attractive and one force repulsive. A symmetrical beginning for the universe with net-zero energy. These appear to be different aspects of the same primordial force.

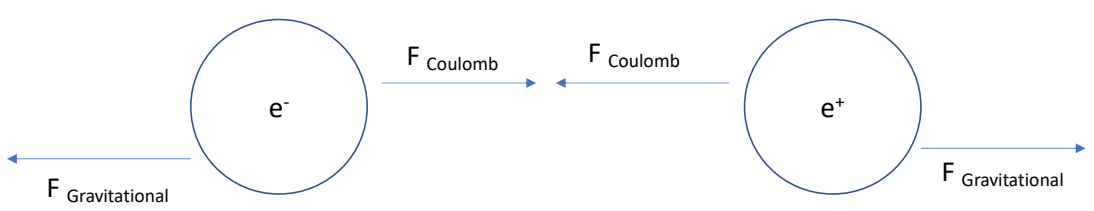

Figure 5.

From a human perspective, labels appeared as in Figure 5. This gives us an understanding of how the Coulomb force and gravitational force are different aspects of the same primordial force. 
We may also observe that the form of the Coulomb force and the Gravitational force are the same:

$$
\begin{aligned}
F_{\text {Coulomb }} & =F_{\text {gravitational }} \\
\frac{Q^{2}}{4 \pi \varepsilon_{0} D^{2}} & =\frac{G_{s} m_{e}^{2}}{D^{2}} \\
\frac{\text { K.Charge }}{D^{2}} & =\frac{\text { K.Mass }^{2}}{D^{2}} \quad K=\text { constant }
\end{aligned}
$$

We find $\mathrm{K}^{2}=(\text { Planck charge })^{-1}$

A symmetrical beginning for the universe with net-zero energy and particles that are mirror images gives positive and negative electromagnetic charges and positive and negative gravitational charges; positive mass for matter and negative mass for antimatter.

\section{Unification Energy}

Since photons can take on energies across the electromagnetic spectrum, it does not make sense to think of unification taking place at a particular energy level. Unification between the Coulomb force and the gravitational force takes place through a variation in the value of the gravitational constant, which is much higher for the strong gravitational force between the electron and the positron.

The composite photon consisting of a positive mass particle and a negative mass antiparticle allows gravity to be combined with the Standard Model of particle physics for the first time.

\section{Range of the Strong Gravitational Force}

The strong gravitational force discovered here is present in all photons. Since the electromagnetic spectrum covers wavelengths ranging from $100,000 \mathrm{~km}$ to 1 picometre, then the force is not microscopic in range but rather operates across a wide range of distances as Newtonian gravity does.

\section{Expansion of Einstein Field Equations to include Vector Gravity}

We can now contemplate the expansion of Einstein's Field Equations to include the strong gravity found here, which is repulsive between positive mass and negative mass. The deep relationship to Coulomb's Law shown here provides the basis for this expansion.

Tentatively, we can say that an equivalence to Maxwell's equations can be developed since we may now view gravity as gravitational charge having positive and negative charges in the same manner as electromagnetism. We may develop Gauss's law for gravity from Newton's law in the same manner that Gauss's law can be developed from Coulomb's law.

Nieto and Goldman $(1991)^{11}$ highlight the possibility of vector gravity for antimatter. Their study concludes that experimental evidence does not exclude this outcome. 
Gauss's law for gravity gives:

$$
\nabla \cdot \mathrm{g}=4 \pi G_{s} \rho
$$

where $\nabla$ is the divergence, $\mathrm{g}$ is the gravitational field and $\rho$ is the mass density. Quantities may be positive or negative.

More generally, we note that Maxwell's equations for electromagnetism may be developed from Coulomb's Law plus the Lorenz invariance transformations of Special Relativity (See "Lagrangian Formulation of the Electromagnetic Field" by Thomas Yu, $16^{\text {th }}$ July $2012^{12}$ ). In a parallel manner, an extended version of Einstein's Field Equations can be developed from Newton's Law of Gravitation plus Special Relativity. This extension will include interactions between the positive and negative gravitational charges and reflect the strong gravitational constant calculated here for the interaction between positive and negative mass.

\section{Conclusion}

A deep relationship is identified between the Coulomb force and Gravity. We demonstrate how this relationship arises through the composite photon. A gravitational constant for strong gravity is calculated from the relationship. The gravitational force is repulsive between matter having positive mass and antimatter having negative mass. Experimental evidence for the composite nature of the photon and for antimatter having negative mass is presented.

The striking equivalence between mass and charge is explored. It is postulated that the Coulomb force and gravity are different aspects of the same primordial force. Implications are given for the expansion of Einstein's Field Equations to include vector gravity.

\section{References:}

1. A Tentative Theory of Light Quanta. Louis de Broglie. Philosophical Magazine Series 6 Volume 47, 1924 - Issue 278

2. Composite Photon Theory versus Elementary Photon Theory. Walton A. Perkins. Journal of Modern Physics Vol. 05 No.18 (2014)

3. Quantum-entangled superluminal double-helix photon produces a relativistic superluminal quantum-vortex zitterbewegung electron and positron. Richard Gauthier. IOP Journal of Physics, Conference Series 1251012016

4. CPT symmetry and antimatter gravity in general relativity. M. Villata. EPL, 94 (2011) 20001

5. Negative Mass in General Relativity. H. Bondi. Rev. Mod. Phys. 29, 423 - Published 1 July 1957

6. Optical diametric drive acceleration through action-reaction symmetry breaking. Wimmer, Regensburger et al. Nature Physics 9, 780-784 (2013) 
7. Precision Mass Spectroscopy of the Antiproton and Proton Using Simultaneously Trapped Particles. Gabrielse et al. Physical Review of Letters Vol. 82, No. 16 April 1999

8. Pair Creation Model of the Universe from Positive and Negative Energy. Choi and Rudra. viXra.org>Relativity and Cosmology>vixRa:1403.0180

9. Virtual gravitational dipoles: The key for the understanding of the Universe? Dragan Hajdukovic. Science Direct: Physics of the Dark Universe, Volume 3, April 2014

10. A unifying theory of dark energy and dark matter:

Negative masses and matter creation within a modified $\Lambda$ CDM framework.

J. S. Farnes. Astronomy and Astrophysics, Volume 620 (December 2018), A\&A, 620 (2018) A92

11. The Arguments Against "Antigravity" and the Gravitational Acceleration of Antimatter. Michael Martin Nieto and T. Goldman. Physics Reports (Review Section of Physics Letters) 205, No. 5 (1991) 221-281. North Holland

12. Lagrangian Formulation of the Electromagnetic Field. Thomas Yu. http://math.uchicago.edu/ may/REU2012/REUPapers/Yu.pdf

13. https://www.researchgate.net/deref/http\%3A\%2F\%2Fdx.doi.org\%2F10.13140\%2FR G.2.2.27190.34884

Negative Mass Solutions to the Dirac Equation Move Forward in Time. Evans Boney. 12 February 2019.

14. Massless states and negative mass states of the coupled electron-positron system with completely symmetric representation of the particles. A. I. Agafonov. ArXiv: 1606.02953 (physics) 20 Jun 2016

15. Negative masses in general relativity and the Dirac equation. F. Winterberg. https://vixra.org/pdf/1201.0081v1.pdf

16. On Negative Mass Black Holes and Supermassive Black Hole Formation. Manuel Uruena Palomo, ResearchGate, May 2020 